\title{
Multiple criteria decision making and economics: an introduction
}

\author{
Rafael Caballero ${ }^{1}$ - Carlos Romero ${ }^{2}$ - Francisco Ruiz ${ }^{1}$
}

Published online: 31 August 2016

CC Springer Science+Business Media New York 2016

In formal terms, the primitive concept characterizing any economic problem is "resource scarcity" within a human behavior context. In other words, the ends tend to be unbounded, so the means are not enough to satisfy them. This epistemic approach to economics as a science, proposed by Lord Robbins in (1932), is today widely accepted, and it can be summarized in the following citation from Robbins:

"Economics is the science which studies human behavior as a relationship between ends and scarce means which have alternative uses".

However, "economic scarcity" in an orthodox analysis is usually formalized by means of rigid constraints such as a budgetary constraint in consumer theory, the technology constraint of a production process, etc. Thus, formally, scarcity is algebraically modeled by a constraint set that defines the feasible solutions of a particular problem. Once the feasible set has been formalized, the preferences of the decision-maker (DM) are introduced with the help of a criterion function such as profits for a producer, utility for a consumer, etc. The embedding of the criterion function and the feasible (constraint) set allows the determination of the optimal solution such as consumer equilibrium, production plan of maximum profit, etc.

This line of thought clearly reduces in logical terms the "economic act" to an "optimization act"; that is, economic problems can be considered formally as particular cases of an optimization problem (e.g., Intriligator 1971, especially chapter 1). In short, orthodox economic rationality consists of maximizing or minimizing a function representing the preferences of the DM subject to the fulfillment of the constraints that define the feasible set.

Rafael Caballero

r_caballero@uma.es

Carlos Romero

carlos.romero@upm.es

Francisco Ruiz

rua@uma.es

1 University of Málaga, Málaga, Spain

2 Technical University of Madrid, Madrid, Spain 
The above approach, which basically underpins all traditional economic problems, is logically sound and internally coherent. However, we do know that, according to basic epistemology, internal coherence is a necessary condition for the acceptance of a scientific framework but it is not a sufficient condition (Popper 1959, pp. 32-33). In other words, the acceptability of a theory requires not only its internal coherence, but also a good level of external coherence or correspondence to the factual reality (i.e., a certain degree of empirical corroboration). From this epistemic perspective, the above theoretical framework for economics presents at least the following two possible drawbacks: (a) In many real situations, the DM preferences are not well characterized by a single criterion function but by a set of conflicting criteria functions of a different nature, and (b) The characterization of the feasible set by rigid constraints that do not allow any type of violation might be unrealistic in many real situations.

These possible shortcomings are clearly due to the optimization theory supporting orthodox economics. However, we should be aware that the science of optimization has dramatically changed in the last 40 years or so. By using the philosophical framework proposed by Thomas Khun (1962), this science has undergone a conceptual revolution leading to a paradigm shift. In fact, the appearance and development of so-called Multiple Criteria Decision Making (MCDM) has opened up new and revolutionary paths for the science of optimization. The following are the basic aspects (that is, the core) of this paradigm. In many cases, DM preferences are not well defined by a single criterion function, but by a set of criteria of a different nature usually in conflict. Besides this, the feasibility of the decisionmaking problem is not necessarily characterized by a set of rigid constraints that rule out any type of violation of the right hand sides. Within the MCDM paradigm, the scarcity leading to the feasible set can be modeled with the help of flexible constraints (e.g., mathematical goals), which allow some type of relaxation; that is, some violations of the right-hand sides of the constraint set are permitted. These violations should be penalized but they do not lead to impossibilities leading to the idea of infeasible solutions.

The MCDM paradigm has been developed mainly as an optimization theory within the discipline of operations research/management science. In this field, MCDM has proved to be a fertile methodology for successfully addressing a wide range of relevant decisionmaking problems. However, the MCDM approach and its underlying philosophy have not been fully incorporated into the core of economic thinking. In short, MCDM remains somewhat unknown in what can be considered orthodox economics. But it would seem to be totally acceptable that if economic problems would be underpinned by the MCDM optimization theory instead of the classic one, at least some of the lack of external coherence problems pointed out above would be considerably mitigated.

In keeping with these ideas, this special volume of the Annals of Operations Research aims at taking a step in the direction of linking economics to MCDM. Thus, we present a collection of papers dealing with the potential effects of solving relevant economic problems by using the tools and the philosophy of the multi-criteria methodology. In this way, two positive results can be obtained: first, the revitalization of orthodox economics with new formulations and analytical procedures taken from the MCDM field; second, the enrichment of the multi-criteria field with the introduction of new problems and areas of application.

The origin of this volume was the 22nd International Conference on Multiple Criteria Decision Making (MCDM 2013) held in Málaga, Spain, in June, 2013. This type of conference is organized every two years by the International Society on MCDM. In the Málaga conference, several streams were devoted in one way or another to dealing with economic problems within the multi-criteria methodology in areas such as business and industrial management, energy, environmental and ecological issues, finance, health and welfare, public 
economics, etc. This volume contains expanded versions of some of the papers presented at the conference, together with other free submissions prepared by researchers who did not attend. We have needed almost three years to coordinate the reviewing process and to complete the volume. We received 42 submissions, among which 20 have been finally accepted for publication after a rigorous reviewing process. In fact, each paper was reviewed following a blind process by at least two referees.

The list and main features of the papers comprising this volume are the following:

- The Paper Multiobjective Project Portfolio Selection with Fuzzy Constraints, by Perez and Gomez, focuses on project portfolio selection and scheduling with multiple criteria within a fuzzy environment by resorting to non-linear binary multiobjective optimization models.

- The paper Fuzzy Portfolio Selection with Non-financial Goals: Exploring the Efficient Frontier, by Calvo et al. also deals with a portfolio selection problem which takes into account classic financial criteria as well as social responsibility criteria, by resorting to fuzzy multi-criteria optimization models.

- The paper An Interactive Approach to Stochastic Programming-Based Portfolio Optimization, by Köksalan and Şakar, is the third paper dealing with portfolio optimization, this time within a stochastic context, by resorting to the interactive technique known as the Weighted Tchebycheff Procedure.

- The paper An Interactive Algorithm to Find the Most Preferred Solution of Multi-Objective Integer Programs, by Lokman et al. is the second paper dealing with multi-criteria interactive methods. In this case, the authors use integer multi-objective programming to investigate the most preferred solution.

- The paper An Interactive Approach for Bi-attribute Multi-item Auctions, by Karakaya and Köksalan, addresses another interactive problem, dealing this time with a bi-attribute, multi-item auction case by considering different structures of preferences of the potential buyer.

- The paper An Extended Goal Programming Model for Site Selection in the Offshore Wind Farm Sector, by Jones and Wall, deals with a problem of offshore wind farm site selection in United Kingdom. The problem is formulated and solved by resorting to an Extended Goal Programming model.

- The paper A Group Decision Making Model Based on Goal Programming with Fuzzy Hierarchy: An Application to Regional Forest Planning, by Bilbao-Terol et al. focuses on group decision making problems in a forest management context. The preferences of the stakeholders are aggregated with the help of fuzzy goal programming models.

- The paper Photovoltaic Power Plants: A Multicriteria Approach to Investment Decisions and a Case Study in Western Spain, by Garcia-Bernabeu et al. deals with a problem related with the construction of photovoltaic plants in Spain. The problem is formulated and solved by resorting to several Compromise Programming models.

- The paper A Participatory Multi-criteria Approach for Power Generation and Transmission Planning, by Bertsch and Fichtner, deals again with the planning of renewable energy, this time in Germany. The approach used jointly considers grid constraints and sensitive multi-criteria decision analysis.

- The paper Stable Solutions for Multiple Scenario Cost Allocation Games with Partial Information, by Borrero et al. deals with the stability of solutions for multiple scenario cost allocation games. The theory is applied to a cost allocation problem of waste collection service in Spain. 
- The paper How Relevant is the Lack of Reciprocity in Pairwise Comparisons? An Experiment with $A H P$, by Linares et al. deals with pairwise comparison matrices where the reciprocity property does not hold. The experiments undertaken seem to indicate that decision makers display significant levels of nonreciprocity, preferring solutions obtained without assuming this property.

- The paper The Precise Consistency Consensus Matrix in a Local AHP-Group Decision Making Context, by Aguarón et al. presents an extension of the Consensus Consistency Matrix Method valid for aggregating pairwise comparison matrices within an AHP context. Some references to applications of the method to real problems appear in the paper.

- The paper Systemic Decision Making in AHP: A Bayesian Approach, by Moreno-Jiménez et al. presents the Systemic Decision Making approach for dealing with complex multiactors decision making problems. The proposed method combines the classic AHP method with Bayesian analysis.

- The paper Selection of Optimum Maintenance Strategy Based on FAHP Integrated with GRA-TOPSIS by Kirubakaran and Ilangkurranran, deals with a problem of maintenance strategy in the paper industry. The proposed method uses fuzzy AHP for determining the preferential weights, and a combination of grey relational analysis and TOPSIS technique for determining the ranking of the four maintenance strategies defined.

- The paper Discrete Multi-Criteria Methods for Lands Use and Conservation Planning on La Colacha in Arroyos Menores (Río Cuarto, Province of Córdoba, Argentina), by Antón et al. deals with a land use planning case study in Argentina, where economic, agronomic, and environmental criteria are jointly considered. The authors use methods based on outranking relationships like ELECTRE and PROMETHEE.

- The paper An ELECTRE Approach for Evaluating Secondary Education Profiles: Evidence from PISA Survey in Serbia, by Stamenković et al. considers the use of an ELECTRE Multi-Level Outranking approach for the assessment of the secondary educational profiles in Serbia. The research uses as secondary source of information the recent PISA report.

- The paper Security Economics: An Adversarial Risk Analysis Approach to Airport Protection, by Cano et al. deals in general with security economics and in particular with the protection of an airport from a terrorist threat. The authors use an analytical tool that derives from the multiattribute expected utility.

- The paper Multiattribute Decision Analysis Using Strict Preference Relations, by Nishizaki et al. proposes a multiattribute utility approach, relaxing the mutual independence condition. In this way, the best alternative through strict preference relations is obtained by solving mathematical programming models.

- The paper Determining Attribute Weights to Improve Solution Reliability and its Application to Selecting Leading Industries, by $\mathrm{Fu}$ and $\mathrm{Xu}$, proposes a method, again within the context of multiattribute decision making, for determining reliable attribute weights. The pros and cons of the method are compared with other methods widely used in the literature.

- The paper Non Additive Robust Ordinal Regression for Urban and Territorial Planning: An Application for Siting an Urban Waste Landfill, by Angilella et al. deals with an urban planning problem in Italy. The authors use the Non Additive Robust Ordinal Regression, which is an extension of the general Robust Ordinal Regression which uses the Choquet integral to quantify the individual preferences.

We would like to thank all the authors for their friendly cooperation and patience in the long review process. We thank all the referees for their generous and invaluable help. Last but not least, we are grateful for the technical help from Katie D'Agosta, Gayathri Balasubramanian, 
and Anandhi Sankar throughout the editorial process as well as the initial and continuous support from the Editor-in-Chief of ANOR, Professor Endre Boros. Their efficient, amicable cooperation has made our work easier and more pleasant.

\section{References}

Intriligator, M. D. (1971). Mathematical optimisation and economic theory. Englewood Cliffs, NJ: PrenticeHall.

Kuhn, T. S. (1962). The structure of scientific revolutions. Chicago: The University of Chicago Press.

Popper, K. R. (1959). The logic of scientific discovery. London: Hutchinson.

Robbins, L. C. (1932). An essay on the nature and significance of economic science. London: Macmillan. 